\title{
Immobilization of Proteins onto the Self-Assembled Phospholipid Layer Fabricated by Plasma-Assisted Method
}

\author{
Shin-ichi Kondo ${ }^{*}$, Yasushi Sasai ${ }^{1}$, \\ Yukinori Yamauchi $^{2}$ and Masayuki Kuzuya ${ }^{2}$ \\ ${ }^{1}$ Laboratory of Pharmaceutical Physical Chemistry, Gifu Pharmaceutical \\ University, 1-25-4 Daigaku-Nishi, Gifu 501-1196, Japan \\ ${ }^{2}$ Department of Pharmaceutical Physical Chemistry, College of \\ Pharmaceutical Sciences, Matsuyama University, 4-2 Bunkyo-cho, Matsuyama, \\ Ehime 790-8578, Japan
}

\section{Keywords: self-assembled phospholipid layer, plasma irradiation, enzyme, low density polyethylene}

\section{Introduction}

We have reported a novel method to introduce a durable surface wettability and minimize its decay with time on several hydrophobic polymers, such as polyethylenenaphthalate, polyethylene, and nylon-12.[1-5] This method involves sorption of methylvinylether-maleic anhydride copolymer (VEMA) into the surface layer and immobilization by a plasma-assisted cross-link reaction. Hydrolysis of VEMA follows to generate carboxyl groups on the surface. Durable surface hydrophilicity introduced in this way has been confirmed both by measurement of the water contact angle and by demonstration of the long-term stability of the surface lubricity on the urethane-made catheter. Several types of bioactive compounds, such as oligo-DNA, heparin, enzyme, and so on, were immobilized onto the durable hydrophilic surface described above. For example, a large amount of oligo-DNA could be immobilized on the durable hydrophilic surface, and the immobilized oligo-DNA could apparently distinguish the complementary oligo-DNA from its single nucleotide polymorphism. However, it was difficult to immobilize enzymes possessing high activity on the durable hydrophilic surface. It is considered that the denaturation of enzymes is caused by the exposure to harsh environment in the course of immobilization and by the interaction with material surface.

We have recently developed a novel fabrication of self-assembled phospholipid (phosphatidyl choline (PC)) layer on hydrophobic polymer surface by plasma-assisted method.[6] The procedure is as follows: (Fig. 1) Alkyl amines, such as hexamethylene diamine (HMDA), were immobilized on the durable hydrophilic film, LDPE-VEMAC film, by condensation reaction to produce LDPE-HE film. LDPE-HE film was immersed into the suspension of PC to fabricate the self-assembled phospholipid layer. The self-assembled phospholipid layer obtained was thermally stable. It is hoped that the selfassembled phospholipid layer would be a good bio-interface between immobilized protein and material surface, because the phospholipid is one of the major components of cytomembrane and would not cause the denaturation of proteins. We also fabricated the self-assembled phospholipid layer incorporating stearic acid (StA), LDPE-StA-PC-SA. StA was used as a scaffold to fix proteins. The model protein, 


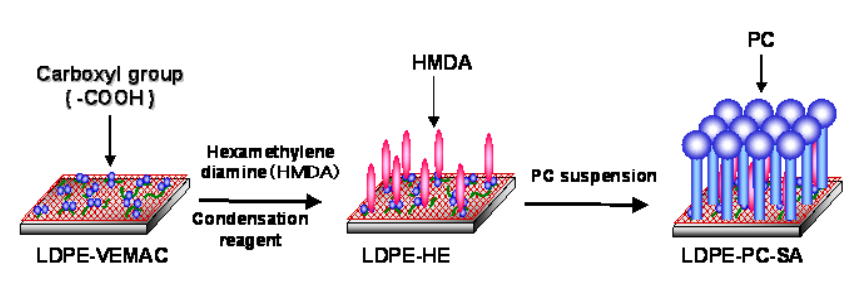

Fig. 1 Schematic illustration for the fabrication of self-assembled phospholipid layer onto a hydrophobic material.

albumin, could be immobilized onto this self-assembled layer. It was also shown that the self-assembled phospholipid layer possessed fluidity.

In the previous papers, we discussed the stability of self-assembled phospholipid layer fabricated by this method.[6,7] It was shown that the stability of self-assembled phospholipid layer depended on the characteristic of grafted alkyl groups acting as anchoring units and the concentration of PC suspension. However, the preparation condition of self-assembled phospholipid layer incorporating StA is not discussed. In this paper, we studied the effect of the concentration of PC suspension containing StA on the thermal stability of self-assembled phospholipid layer. We immobilized three kinds of enzymes as model proteins on the selfassembled phospholipid layer incorporating StA. The surface density of immobilized protein was estimated. The activity of model enzyme, $\beta$-galactosidase, was also estimated by the decomposition of $o$-nitrophenyl- $\beta$-D-galactopyanoside.

\section{Experimental}

2.1 Fabrication of self-assembled phospholipid layer incorporating stearic acid

The LDPE-VEMAC film was prepared according to the method reported previously.[6] Hexamethylene diamine (HMDA) was introduced into LDPE-VEMAC film by the condensation reaction. The LDPE film grafting HMDA was soaked into various concentration of phosphatidyl choline (PC) suspension containing stearic acid (StA) $(10 \mathrm{ml})$ at $30{ }^{\circ} \mathrm{C}$ for $24 \mathrm{~h}$. The ratio of StA against PC was constant (PC:StA=1:6). The film was washed with water, and dried in vacuo to obtain LDPE-StA-PC-SA film.

\subsection{Thermal stability of LDPE- StA-PC-SA film}

The LDPE-StA-PC-SA film was soaked into $5 \mathrm{ml}$ water. The amount of $\mathrm{PC}$ eluted from LDPE-StA-PC-SA film was measured by UV spectrometer $(210 \mathrm{~nm})$ at various temperatures.

\subsection{Immobilization of model proteins onto LDPE-StA-PC-SA film}

We selected three kinds of enzymes possessing different molecular weight, acetylcholineesterase (ACHE, $280 \mathrm{kDa}$ ), $\beta$-galactosidase (BG, 116 $\mathrm{kDa}$ ) and peroxidase (POD, $44 \mathrm{kDa}$ ), as model proteins. The LDPE-StA-PC-SA film was soaked into $10 \mathrm{mM}$ phosphate buffer $(\mathrm{pH} 4.0,5$ $\mathrm{ml}$ ) containing $48 \mathrm{mg}$ of 1-ethyl-3-(3-dimethylaminopropyl)carbodiimide hydrochloride (EDC) at room temperature for $2 \mathrm{~h}$. This film was washed with water. The film was immersed into $10 \mathrm{ml}$ of various concentrations of model proteins (from 10 to $200 \mu \mathrm{g} / \mathrm{ml}$ ) in phosphate buffered

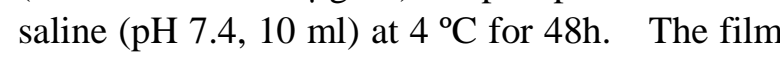
obtained was washed with a phosphate buffered saline (PBS, pH 7.4).

The amount of immobilized proteins was measured by Bradford method.[8] The unreacted proteins in PBS was reacted with coomassie brilliant blue (CBB) solution (protein assay CBB solution), and the absorbance at 450 nm was measured.

2.4 Activity estimation of BG immobilized onto LDPE-StA-PC-SA film

$60 \mathrm{mmol} / \mathrm{l} \quad o$-Nitrophenyl- $\beta$-D-galactopyranoside (ONPG, $600 \mu \mathrm{l}), 1.4 \mathrm{~mol} / \mathrm{l}$ 2-mercaptoethanol $(100 \mu \mathrm{l}), 15 \mathrm{mmol} / \mathrm{l}$ magnesium chloride $(100 \mu \mathrm{l})$ and PBS $(8.2 \mathrm{ml})$ were added to the LDPE-StA-PC- SA film immobilizing BG. This solution was kept at $40{ }^{\circ} \mathrm{C}$. The film was removed from the solution, and then the absorbance at $405 \mathrm{~nm}$ was measured. (Fig. 2)

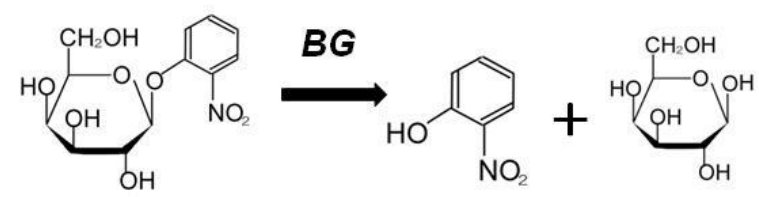

o-Nitrophenyl- $\boldsymbol{\beta}$ -

D-galactopyranoside

o-Nitrophenol

Fig. 2 Scheme of enzymatic cleavage of $o$-nitrophenyl- $\beta$-D-galactopyranoside. 


\section{Results and Discussion}

3.1 Effect of the concentration of PC suspension containing StA on the thermal stability of LDPEStA-PC-SA film

Figure 3 shows the elution ratio of $\mathrm{PC}$ from the self-assembled phospholipid layer incorporating StA (LDPE-StA-PC-SA) film against temperature. The ratio of StA against $\mathrm{PC}$ was constant (PC:StA=1:6). In this experimental condition, the most thermally stable film was obtained in the case of $1 \mathrm{mM}$ PC suspension, but PC was gradually eluted from $60{ }^{\circ} \mathrm{C}$. We have reported that LDPE-PC-SA, which does not contain StA, is thermally stable up to $80{ }^{\circ} \mathrm{C}$.[7] It is also well-known that the fluidity of cytomembrane increases with the increase of fatty acid in cytomembrane. Therefore, it is considered that the incorporation of StA caused the thermal instability, which would relate to the fluidity of self-assembled phospholipid layer. The selfassembled phospholipid layer became more unstable with the increase of the concentration of PC suspension. It is assumed that excess amount of PC adsorbed on the film would be eluted. $0.5 \mathrm{mM}$ PC suspension also produced the unstable self-assembled phospholipid layer. It is considered that thermally stable selfassembled phospholipid layer would not be fabricated due to insufficient amount of PC.

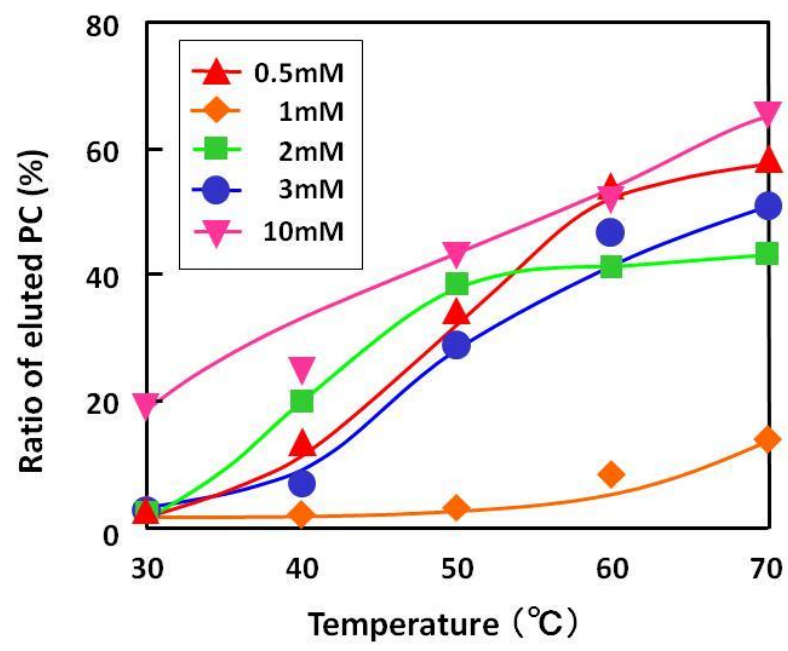

Fig.3 Plots of the elution ratio of $\mathrm{PC}$ from LDPE-StA-PC-SA against temperature.
3.2 Immobilization of model proteins on LDPE-StA-PC-SA film

Three kinds of enzymes possessing different molecular weight, acetylcholineesterase (ACHE, $280 \mathrm{kDa}), \beta$-galactosidase (BG, $116 \mathrm{kDa})$ and peroxidase (POD, $44 \mathrm{kDa}$ ), were immobilized on LDPE-StA-PC-SA film. Figure 4 shows plots of the surface density of immobilized enzymes against the amount of used enzymes. Although the surface density of immobilized enzyme increased with the increase of the amount of used enzyme, the surface density gradually tended to level off in each enzyme. It was also shown that the maximum value of surface density in each enzyme tended to be inversely proportional to the molecular weight within this experimental condition.

3.3 Activity estimation of $\beta$-galactosidase immobilized onto self-assembled phospholipid layer

We studied the activity of BG immobilized onto LDPE-StA-PC-SA film. $o$-Nitrophenyl- $\beta$ D-galactopyranoside (ONPG) was used as a substrate of BG. ODPG was enzymatically cleaved by BG to produce $o$-nitrophenol. The absorbance of produced $o$-nitrophenol was measured by UV spectrophotometer.

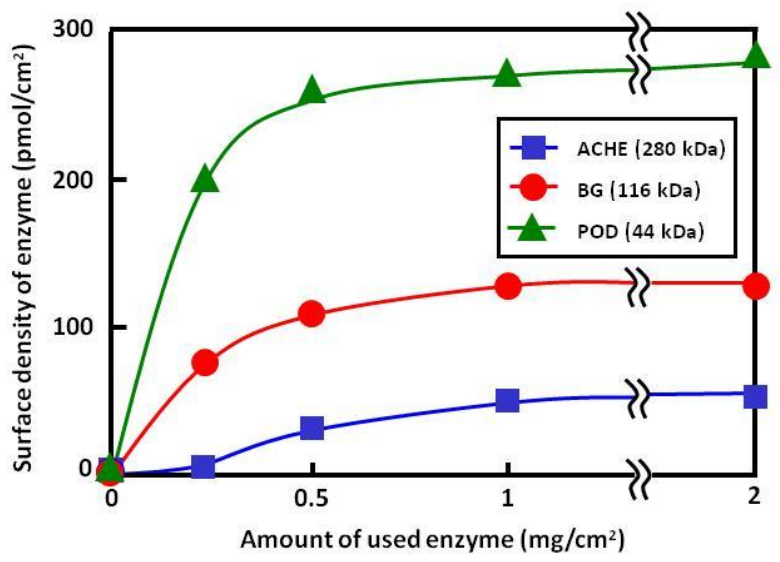

Fig.4 Plots of the surface density of immobilized enzymes against the amount of used enzymes. 
Table 1 shows the density and the specific activity of BG immobilized on LDPE-StA-PC-SA film (LDPE-PC-BG) together with those results of BG immobilized on LDPE-VEMAC film (LDPE-BG). The density of immobilized BG was almost the same in each sample, but the specific activity of LDPE-PC-BG was about 2.5 times as high as that of LDPE-BG. This result suggests that the self-assembled phospholipid layer would act as a good bio-interface between enzyme and material surface.

Table 1 Density and Specific Activity of BG

\begin{tabular}{lcc}
\hline & $\begin{array}{c}\text { Density of BG } \\
\left(\mathrm{pmol} / \mathrm{cm}^{2}\right)\end{array}$ & $\begin{array}{c}\text { Specific activity of BG } \\
(\mathrm{U} / \mu \mathrm{g})\end{array}$ \\
\hline LDPE-PC-BG & 102 & 86 \\
LDPE-BG & 129 & 32 \\
\hline & $\begin{array}{l}\text { LDPE-PC-BG: BG immobilized on LDPE-StA-PC-SA film } \\
\text { LDPE-BG: BG immobilized on LDPE-VEMAC film }\end{array}$
\end{tabular}

\section{Conclusion}

The conclusions drawn from the present study can be summarized as follows.

The thermal stability of the self-assembled phospholipid layer incorporating stearic acid depended on the concentration of PC suspension. Three kinds of model enzymes were immobilized on LDPE-StA-PC-SA film. The maximum value of surface density of enzyme tended to be inversely proportional to the molecular weight. We also studied the activity of BG immobilized onto LDPE-StA-PC-SA film. The specific activity of BG immobilized on LDPE-StA-PC-
SA was higher than that of BG immobilized on LDPE-VEMAC. It was suggested that the self-assembled phospholipid layer would act as a good bio-interface.

We are now actively elaborating the application of biosensor using LDPE-StA-PC-SA film.

\section{Acknowledgements}

This work was financially supported in part by a Grant in Aid for Scientific Research (B) from the Ministry of Education, Culture, Sports, Science and Technology of Japan (Grant no. 22390009), which is gratefully acknowledged.

\section{References}

1. M. Kuzuya, T. Yamashiro, J. Photopolym. Sci. Technol. 8 (1995) 381.

2. M. Kuzuya, Y. Matsuno, T. Yamashiro, M. Tuiki, Plasmas and Polymers 2 (1997) 79.

3. M. Kuzuya, T. Yamashiro, S. Kondo, M. Tuiki, Plasmas and Polymers 2 (1997) 133.

4. M. Kuzuya, T. Sawa, T. Yamashiro, S. Kondo, O. Takai, J. Photopolym. Sci. Technol. 14 (2001) 87.

5. S. Kondo, Y. Sasai, M. Kuzuya, Thin Solid Films 515 (2007) 4136.

6. S. Kondo, Y. Sasai, Y. Yamauchi, M. Kuzuya, J. Photopolym. Sci. Technol. 22 (2009) 477

7. S. Kondo, Y. Sasai, Y. Yamauchi, M. Kuzuya, $J$. Photopolym. Sci. Technol. 23 (2010) 567

8. M. M. Bradford, Anal. Biochem. 72 (1976) 248 